\title{
Time Delay Compensation in Power Controlled Cellular Radio Systems
}

\author{
Fredrik Gunnarsson, Fredrik Gustafsson \\ Division of Communication Systems \\ Department of Electrical Engineering \\ Linköpings universitet, SE-581 83 Linköping, Sweden \\ WWW: http://www.comsys.isy.liu.se \\ Email: fred@isy.liu.se, fredrik@isy.liu.se
}

5th February 2001

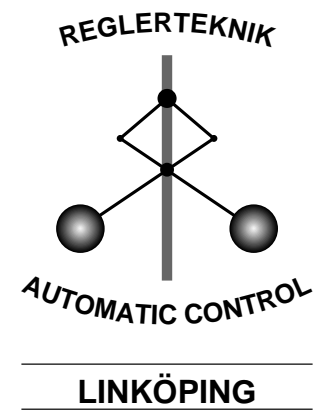

Report No.: LiTH-ISY-R-2334

Submitted to IEEE Communications Letters

Technical reports from the Communication Systems group in Linköping are available by anonymous ftp at the address ftp.control.isy.liu.se. This report is contained in the file 2334.pdf. 


\begin{abstract}
Transmission power control is an important means to manage the radio resources in wireless communications. The performance, however, may be reduced due to time delays as in any controlled system. Most controllers to date are designed without considering time delays, which may result in oscillatory or unstable systems. To overcome these problems, Time Delay Compensation (TDC) is introduced. The main idea is to adjust the measurements (e.g. SIR) to include the effects of issued but not yet applied power control commands. TDC thereby improves the stability and performance of an arbitrary power control algorithm. The benefits are illustrated by simulations using some popular power control algorithms.
\end{abstract}

Keywords: Power control, Wireless networks, Time delays, Delay compensation, DS-CDMA, GSM 


\title{
Time Delay Compensation in Power Controlled Cellular Radio Systems
}

\author{
Fredrik Gunnarsson and Fredrik Gustafsson
}

\begin{abstract}
Transmission power control is an important means to manage the radio resources in wireless communications. The performance, however, may be reduced due to time delays as in any controlled system. Most controllers to date are designed without considering time delays, which may result in oscillatory or unstable systems. To overcome these problems, Time Delay Compensation (TDC) is introduced. The main idea is to adjust the measurements (e.g., SIR) to include the effects of issued but not yet applied power control commands. TDC thereby improves the stability and performance of an arbitrary power control algorithm. The benefits are illustrated by simulations using some popular power control algorithms
\end{abstract}

\section{INTRODUCTION}

Closed-loop power control is widely discussed as a means to maintain acceptable quality of service in cellular radio systems. The objective is to compensate for varying channel gains and to control the multiple access interference (MAI). A common strategy is to control each link individually and distributedly, based only on local information. An outer loop provides an inner loop with a target value (e.g., target SIR). The inner loop then issues transmission powers or transmission power control commands to meet this target.

This works well under ideal assumptions, but in real situations, time delays in the control loops reduce the performance. These delays are typically round-trip delays in the inner loop. As concluded in [1], the delays result in oscillative and possibly unstable systems. This in turn affect the spectral efficiency of the system [2].

In Section III in this Letter, we propose a general technique, referred to as Time Delay Compensation (TDC), to compensate for the delays. The technique can be used together with a wide range of power control algorithms, as described in a general framework in Section II. To exemplify its merits, it is applied to two popular power control algorithms in illuminating simulations in Section IV, followed by some concluding remarks.

\section{System Model}

It will be assumed that each mobile station is connected to only one base station, and soft handover schemes are discussed in the end of Section III. Moreover, the nomenclature is settled to focus on the uplink (reverse link). However, the downlink (forward link) is treated analogously.

\section{A. Notation}

All values will be represented by values in logarithmic scale (e.g., $\mathrm{dB}$ or $\mathrm{dBW}$ ). The $m$ active mobile stations are transmitting using the powers $p_{i}(t), i=1, \ldots, m$. Let $i$ identify each link, so that e.g., receiver $i$ is located in the base station to which mobile station $i$ is connected. The signal from mobile station $i$ to receiver $j$ is attenuated by the power gain $g_{i j}(t)(<0)$. Thus receiver $i$ will experience a desired carrier power

$$
C_{i}(t)=p_{i}(t)+g_{i i}(t)
$$

and an interference plus noise $I_{i}(t)$. The signal-to-interference ratio $(\mathrm{SIR}) \sqrt[1]{1}$ at base station $i$ is defined by

$$
\gamma_{i}(t)=p_{i}(t)+g_{i i}(t)-I_{i}(t)
$$

Processing and signaling takes time, introducing time delays in the control loops. Due to specified signaling protocols, it is relevant to describe time delays in terms of the sampling interval or power update interval. Hence, the delayed signals can be described using the delay operator $q$, defined by

This work was supported by the Swedish National Board for Industrial and Technical Development (NUTEK) and has been carried out in cooperation with Ericsson Radio Systems AB, which both are acknowledged.

${ }^{1}$ Sometimes the term carrier-to-interference ratio $(\mathrm{C} / \mathrm{I})$ is used. The actual difference is whether the processing gain $(\mathrm{PG})$ is included in the interference or not. From a dynamical point of view, this is irrelevant. 


$$
q^{-n} p_{i}(t)=p_{i}(t-n)
$$

Assume that issued powers or power control commands are delayed by $n_{p}$ samples before they take effect. Furthermore, assume that a measurement is used in the control algorithm $n_{m}$ samples after it is collected (for example if the measurement is sent over the radio interface). Furthermore, the power control algorithm iteself includes a processing delay of one sample. A typical situation is $n_{p}=1$ and $n_{m}=0$, which will be used in the simulations.

\section{B. Power Control Algorithms}

Two popular power control algorithms will be described below, together will a generic description of a general power control algorithm. In the emerging WCDMA-standards, up/down power control will be employed 3 .

$$
\begin{array}{ll}
\text { Receiver: } & s_{i}(t)=\operatorname{sign}\left(\gamma_{\mathrm{i}}^{\mathrm{t}}(\mathrm{t})-\gamma_{\mathrm{i}}(\mathrm{t})\right) \\
\text { Transmitter: } & p_{i}(t+1)=p_{i}(t)+\Delta_{i} s_{i}(t)
\end{array} \Longleftrightarrow p_{i}(t)=\frac{\Delta_{i}}{q-1} \operatorname{sign}\left(\gamma_{i}^{t}(t)-\gamma_{i}(t)\right),
$$

where $\gamma_{i}^{t}(t)$ is the target value provided by an outer loop. The algorithm will be referred to as Fixed-Step Power Control, (FSPC). Taking the delays into consideration, the local dynamics of this link can be depicted as in Figure 1] A similar algorithm is the Distributed Power Control (DPC) algorithm [4], [1]

$$
p_{i}(t+1)=p_{i}(t)+\left(\gamma_{i}^{t}(t)-\gamma_{i}(t)\right) \Longleftrightarrow p_{i}(t)=\frac{1}{q-1}\left(\gamma_{i}^{t}(t)-\gamma_{i}(t)\right)
$$

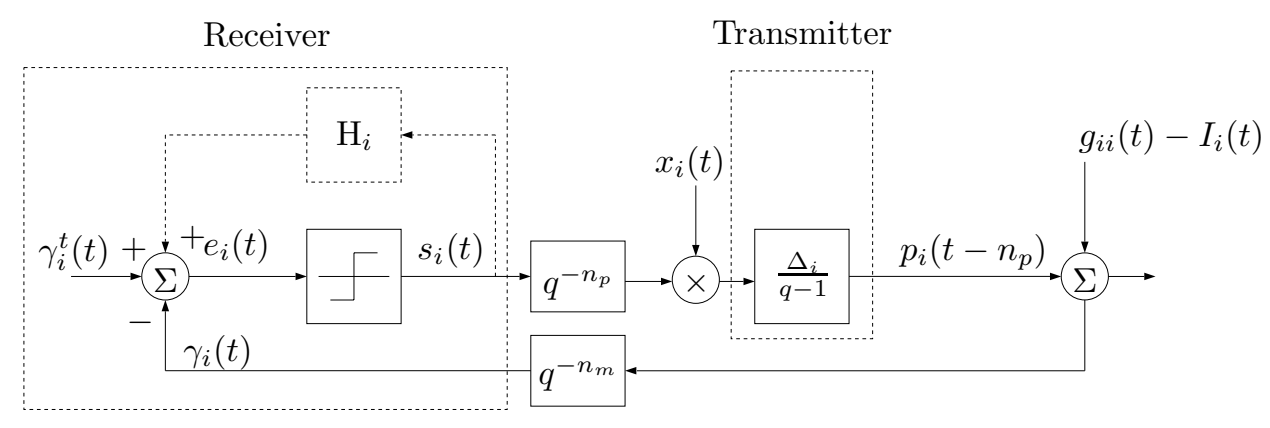

Fig. 1

LOCAL DYNAMicS OF THE WCDMA UP/DOWN POWER CONTROL ALGORITHM (FSPC). The POWER CONTROL

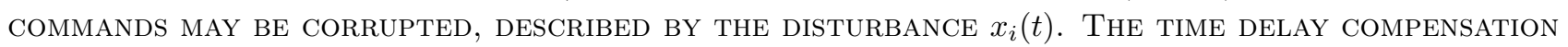
IMPLEMENTATION IS DESCRIBED BY THE PROCESS $\mathrm{H}_{i}$.

In a general and generic setting, the issued powers or control commands depend on measurements, normally either SIR or received carrier power. These measurements, denoted $m_{i}(t)$, may be filtered by a filtering operation $\mathrm{F}_{i}$ (assumed additive and time-invariant). The filtered measurements (outdated by $n_{m}$ samples) together with corresponding target values $m_{i}^{t}(t)$ from an outer loop are used in the control mechanism $\mathrm{R}_{i}$ (resulting in a time delay of one sample) to issue power control commands $s_{i}(t)$, which are sent over the radio interface. The control commands (outdated by $n_{p}$ samples) are decoded by the device $\mathrm{D}_{i}$ on the transmitter side into updated transmission powers $p_{i}(t+1)$. Note that $\mathrm{D}_{i}$ include physical constraints, such as limited dynamic range and quantization. This generic case is depicted in Figure 2,

\section{Time Delay Compensation}

The main idea is to adjust the measurements to also reflect issued commands that not yet have taken effect. In practice this involves monitoring the power to be used. This can be formalized for the generic case in the previous section as the following algorithm 


\section{Algorithm: Time Delay Compensation (TDC)}

i) Adjust measurements: $\tilde{m}_{i}(t)=m_{i}(t)+\mathrm{F}_{i}\left\{\tilde{p}_{i}(t)\right\}-\mathrm{F}_{i}\left\{\tilde{p}_{i}\left(t-n_{p}-n_{m}\right)\right\}$.

ii) Issue power control command: $s_{i}(t)=\mathrm{R}_{i}\left\{m_{i}^{t}(t), \tilde{m}_{i}(t)\right\}$.

iii) Monitor output powers to be used: $\tilde{p}_{i}(t+1)=\mathrm{D}_{i}\left\{s_{i}(t)\right\}$.

Schematically, this is implemented as in Figure 2, where $\mathrm{H}_{i}$ represents the time delay compensation as described above.

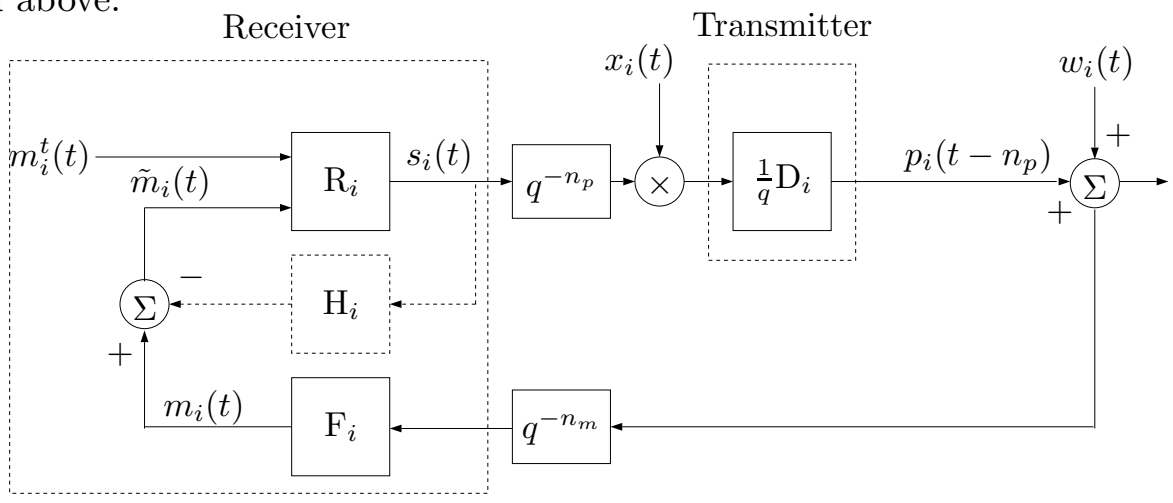

$$
\begin{aligned}
& \text { Carrier - based : } \\
& m_{i}(t)=C_{i}(t), \\
& w_{i}(t)=g_{i i}(t) \\
& \text { SIR - based : } \\
& m_{i}(t)=\gamma_{i}(t), \\
& w_{i}(t)=g_{i i}(t)-I_{i}(t)
\end{aligned}
$$

Fig. 2

BLOCK DIAGRAM OF A RECEIVER-TRANSMITTER PAIR WHEN EMPLOYING A GENERAL POWER CONTROL ALGORITHM With THE OPTIONAL TIME DELAY COMPENSATION $\left(\mathrm{H}_{i}\right)$. BOTH CARRIER POWER BASED AND SIR-BASED POWER CONTROL ARE COVERED.

For a direct example, consider the FSPC algorithm in (1), no measurement filtering and the typical case $n_{m}=0, n_{p}=1$. In this simple case step $i$ ) and $\left.i i i\right)$ can be combined and the algorithm reduces to

\section{Algorithm: Time Delay Compensation (TDC) (WCDMA specific case)}

i) Adjust measurements: $\tilde{\gamma}_{i}(t)=\gamma_{i}(t)+\Delta_{i} s_{i}(t-1)$.

ii) Issue power control command: $s_{i}(t)=\operatorname{sign}\left(\gamma_{i}^{t}(t)-\tilde{\gamma}_{i}(t)\right)$.

Time Delay Compensation is applicable, where the power control algorithm can be described by the generic case. However, when in soft handover, the mobile considers only one of the power control commands. Therefore, TDC should be disabled while the mobile is in soft handover. Further details on power control and TDC are provided in [5].

\section{Simulations}

To illuminate the stabilizing effect of TDC, a simple case is considered. Five mobiles (mean vel. $5 \mathrm{~m} / \mathrm{s}$, subject to shadow fading, corr. dist. $110 \mathrm{~m}, \sigma=6 \mathrm{~dB}$ ) are supported by a rural cell plan. The adaption of the FSPC and DPC algorithms (Section II-B) are illustrated by Figure 3. DPC is unstable when subject to delays 1]. TDC is seen to stabilize DPC and to reduce the oscillatory behavior of the FSPC algorithm. This is interesting, since more stable operation implies that less fading margin is needed, which in turn improves the spectral efficiency.

\section{Conclusions}

Time Delay Compensation (TDC) is proposed, to reduce the effects of round-trip delays in power-controlled cellular radio systems. The technique can be employed together with almost any power control algorithms based on either carrier power or SIR measurements. The main idea is to adjust the measurements to also reflect issued commands that not yet have taken effect. Simulations, using the up/down algorithm in WCDMA and the Distributed Power Control (DPC) algorithm, illustrates the stabilizing effect of TDC. 

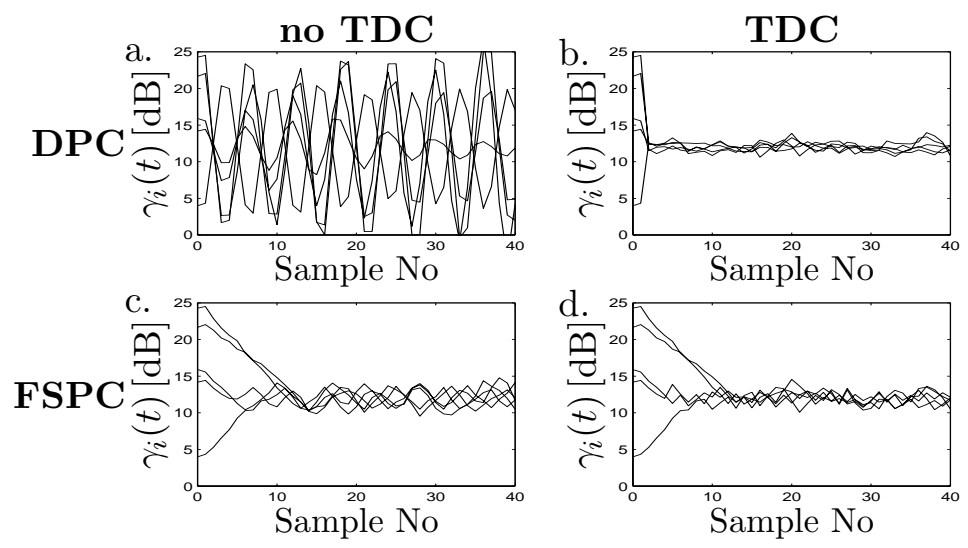

Fig. 3

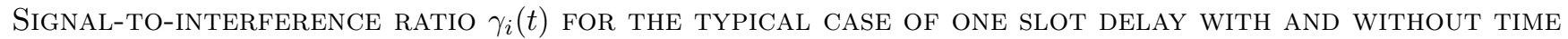
Delay COMPensation (TDC). The target Value in All CASES IS $\gamma_{i}^{t}(t)=12$ DB.

\section{REFERENCES}

[1] F. Gunnarsson, J. Blom, and F. Gustafsson. Power control in cellular systems subject to constraints and time delays. In Proc. IEEE Global Telecommunications Conference, Sydney, Australia, November 1998.

$[2]$ J.H. Jr. Gass, D.L. Noneaker, and M.B. Pursley. Spectral efficiency of a power-controlled CDMA mobile personal communication system. IEEE Journal on Selected Areas in Communications, 14(3), April 1996.

[3] S. Ariyavisitakul. Signal and interference statistics of a CDMA system with feedback power control - part II. IEEE Transactions on Communications, 42(2/3/4), 1994.

[4] G.J. Foschini and Z. Miljanic. A simple distributed autonomus power control algorithm and its convergence. IEEE Transactions on Vehicular Technologu. 42(4). 1993.

[5] F. Gunnarsson. Power Control in Cellular Radio System: Analysis, Design and Estimation. PhD thesis, Linköpings universitet, Linköping, Sweden, April 2000. Available at http://www.control.isy.liu.se. 